\title{
Ubiquitin-Specific-Processing Protease 7 Regulates Female Germline Stem Cell Self-Renewal Through DNA Methylation
}

\author{
Yongqiang Zhao ${ }^{1} \cdot$ Xiaoyong $\mathrm{Li}^{1} \cdot$ Geng Tian ${ }^{1} \cdot$ Xinyan $\mathrm{Zhao}^{2} \cdot \mathrm{Jiemin}_{\mathrm{Wong}}{ }^{3} \cdot$ Yue Shen $^{2} \cdot \mathrm{Ji} \mathrm{Wu}^{1,2}$ (I)
}

Accepted: 27 October 2020 / Published online: 5 November 2020

(C) The Author(s) 2020

\begin{abstract}
Ubiquitin-specific-processing protease $7\left(U_{s p} 7\right)$ is a key deubiquitinase controlling epigenetic modification and regulating the self-renewal, proliferation, and differentiation of stem cells. However, the functions and mechanisms of action of Usp 7 on female germline stem cells (FGSCs) are unknown. Here, we demonstrated that Usp 7 regulated FGSC self-renewal via DNA methylation. The results of Cell Counting Kit-8 and 5-ethynyl-20-deoxyuridine assays showed that the viability and proliferation of FGSCs were negatively regulated by Usp 7 . Moreover, Usp 7 downregulated the expression of self-renewal genes, such as Oct4, Etv 5, Foxol, and Akt, but upregulated the expression of differentiation-related genes including Stra 8 and Sycp3. Mechanistically, RNA-seq results showed that $U s p 7$ negatively regulated FGSC self-renewal but positively modulated differentiation in FGSCs. Meanwhile, both overexpression and knockdown of Usp 7 resulted in significant changes in DNA methylation and histone modification in FGSCs. Additionally, RNA-seq and MeDIP-seq analyses showed that $U s p 7$ regulates the self-renewal and differentiation of FGSCs mainly through DNA methylation rather than histone modification, which was also confirmed by a rescue assay. Our study not only offers a novel method to research FGSC self-renewal and differentiation in view of epigenetic modifications, but also provides a deep understanding of FGSC development.
\end{abstract}

Keywords Usp 7 FGSCs $\cdot$ DNA methylation $\cdot$ Histone modification $\cdot$ Self-renewal $\cdot$ Differentiation

\section{Introduction}

It has been reported that about 48.5 million couples suffer from infertility worldwide [28]. Disorders in oogenesis are

These authors "Yongqiang Zhao, Xiaoyong Li, Geng Tian, Xinyan Zhao" contribute equally to this work

Supplementary Information The online version contains supplementary material available at https://doi.org/10.1007/s12015-02010076-9.

$\mathrm{Ji} \mathrm{Wu}$

jiwu@sjtu.edu.cn

1 Renji Hospital, Key Laboratory for the Genetics of Developmental and Neuropsychiatric Disorders (Ministry of Education), Bio-X Institutes, School of Medicine, Shanghai Jiao Tong University, Shanghai 200240, China

2 Key Laboratory of Fertility Preservation and Maintenance of Ministry of Education, Ningxia Medical University, Yinchuan 750004, China

3 Shanghai Key Laboratory of Regulatory Biology, Fengxian District Central Hospital-ECNU Joint Center of Translational Medicine, Institute of Biomedical Sciences and School of Life Sciences, East China Normal University, Shanghai 200241, China the main cause of female infertility. Oogenesis is a highly complex process that is intricately regulated by the interactions of multiple genes and various signaling molecules, which makes the investigation of oogenesis disorders extremely challenging. Many risk factors, such as genetic, epigenetic, and environmental ones, result in oogenesis disorders. In recent years, the discovery of female germline stem cells (FGSCs) has provided a new perspective for the treatment of such diseases $[41,42,50]$. As the germline stem cells that supplement oocytes, FGSCs can maintain the reproductive function of the ovary, indirectly maintain the endocrinological status of the ovary, and delay premature ovarian failure. They are of great significance for improving the quality of follicles and the pregnancy rate of female mammals and are becoming a particular focus of the medical community.

Several studies have revealed the mechanism underlying FGSC self-renewal, differentiation, and autophagy [19, 46, 51, 52]. Additionally, FGSCs were demonstrated to have the potential to differentiate into oocytes, which provided a new strategy to research oogenesis [7, 41, 52]. Comparing FGSCs and spermatogonial stem cells (SSCs), Li et al. uncovered the transcript structures, genetic variants, and interaction between microRNAs and circRNAs [20]. Li et al. suggested that 
chemical compounds play important roles in modulating FGSCs via the PI3K/Akt pathway [19]. Additionally, Ma et al. revealed that Etv5, Foxol, and Akt genes positively regulate FGSC self-renewal [27]. Epigenetic research on the development of stem cells, especially germ stem cells, has become a hotspot and a field in which many outstanding results were achieved $[12,18]$. Zhang et al. revealed that DNA methylation contributes to the unipotency of FGSCs and is involved in the maintenance of FGSC sexual identity [49]. These studies established the theoretical foundations for researching the regulatory mechanism of FGSCs and provided a deep understanding of germ cell development.

Ubiquitin-specific-processing protease $7\left(U_{s p} 7\right)$ is a wellknown deubiquitinase. It is widely expressed and works by stabilizing substrate proteins [31,34]. Usp 7 removes ubiquitin from multiple protein substrates and participates in a variety of cellular processes, including immune response, mitosis, and DNA repair $[1,2,24,30,34]$. Recent studies have shown that $U_{s p} 7$ is involved in cell differentiation. For example, it is required for the osteogenic differentiation of human adipose-derived stem cells [36]. Additionally, Liang et al. revealed that $U s p 7$ regulates human terminal erythroid differentiation [22] and promotes the invasion and metastasis of cancer [25]. Deubiquitination is the basic function of $U s p 7$ and its epigenetic regulation has become a new research hotspot. USP7 participates in the regulation of methylation through interacting with DNMT1, DNMT3a, DNMT3b, and UHRF1 $[4,9]$. Additionally, Usp 7 had a major effect on the progress of histone modification $[23,40]$. Histone modification is an important way in which $U_{s p} 7$ regulates cellular functions $[10,37,38]$. Usp 7 is now a key molecule in epigenetic research and has been a focus of ongoing studies. However, to the best of our knowledge, no studies have evaluated the mechanism and function of Usp 7 in determining FGSC selfrenewal and differentiation.

In this paper, we report for the first time that $U s p 7$ inhibited the proliferation of FGSCs and promoted their differentiation via genomic methylation. This study provides a better understanding of the regulatory mechanism of FGSC self-renewal and differentiation, and might provide a theoretical basis for improving the diagnosis and treatment of ovarian dysfunction and other related diseases.

\section{Results}

\section{Characterization of Female Germline Stem Cells}

To characterize the FGSCs used in this study (Fig. 1a), we identified marker genes of germ cells: Oct4 (also known as Pou5f1, POU domain, class 5, transcription factor 1) [32], $M v h$ [also known as $D d x 4$, DEAD (Asp-Glu-Ala-Asp) box polypeptide 4] [6], Blimpl (also known as prdml, PR domain containing 1) [29], and Dazl (deleted in azoospermia-like)
[45]. The expression of Oct4, Mvh, Blimpl, and Dazl was confirmed by RT-PCR (Fig. 1b). The positivity of MVH was further identified by immunofluorescence analysis (Fig. 1c). These obtained results suggested that the FGSCs used were standardized.

\section{Usp7 Is Upregulated during the Differentiation of Female Germline Stem Cells Induced by Retinoic Acid and Granulosa Cells}

To study the involvement of $U s p 7$ in the development of FGSCs, we first examined its expression in the ovary of postnatal mice at various times $(3,7,21$, and 42 days) by qRTPCR and western blotting (Fig. 2a, b). The results showed that the expression of $U s p 7$ gradually increased in the process of ovarian development. This indicated that $U s p 7$ plays an important role in FGSC development. qRT-PCR and western blotting were then performed to analyze the expression of Usp 7 during the in vitro differentiation of FGSCs induced by retinoic acid and granulosa cells [7, 52]. The results showed that the expression of $U s p 7$ was increased in differentiated FGSCs (Fig. 2c-2f). We deduced that Usp 7 is upregulated during the differentiation of FGSCs.

\section{Knockdown of Usp7 Enhances Female Germline Stem Cell Growth and Self-Renewal}

To further investigate the function of $U s p 7$ in FGSCs, we knocked down Usp7 using shRNAs (Fig. 3a). The results of Usp 7 knockdown in FGSCs were confirmed by qRT-PCR and western blotting. Knockdown of $U_{s p} 7$ resulted in more than $75 \%$ and $50 \%$ decreases in mRNA (Fig. 3b) and protein levels (Fig. $3 \mathrm{c})$, respectively, compared with those in the control. We next examined the effect of $U s p 7$ knockdown on the proliferation of FGSCs using CCK8 assay (Fig. 3d) and EdU incorporation assay (Fig. 3e). The results showed that knockdown of $U_{s p} 7$ in FGSCs significantly improved their proliferation compared with that of controls. Accordingly, the expression of Oct4, Etv5, Foxol, and Akt, genes related to FGSC self-renewal, was upregulated in Usp7-knockdown FGSCs (Fig. 3f). Meanwhile, Stra8 and Sycp3, differentiation-related genes, showed no significant changes in Usp 7-knockdown FGSCs compared with the levels in the corresponding control (Fig. 3g). These results together indicate that the knockdown of Usp 7 enhanced FGSC growth and self-renewal.

\section{Usp7 Overexpression Inhibits Female Germline Stem Cell Growth and Self-Renewal, while Improving Female Germline Stem Cell Differentiation}

We next investigated how upregulated Usp 7 affects the growth and self-renewal of FGSCs by overexpressing $U s p 7$. The FGSC line was transfected with lentivirus expressing control vector and 
Fig. 1 Characteristics of female germline stem cells. (a)

Representative microscopy images of the morphological characteristics of female germline stem cells (FGSCs). (b) Reversetranscription PCR identification of Mvh, Oct4, Blimpl, and Dazl mRNA expression in FGSCs. M, 250 bp DNA markers. Gapdh serves as an endogenous control for normalization. $N C$ negative control. (c) Immunofluorescence staining of MVH in FGSCs $(n=3$ per group). Scale bars: $10 \mu \mathrm{m}$ in (a) and (c) $\mathbf{a}$

b
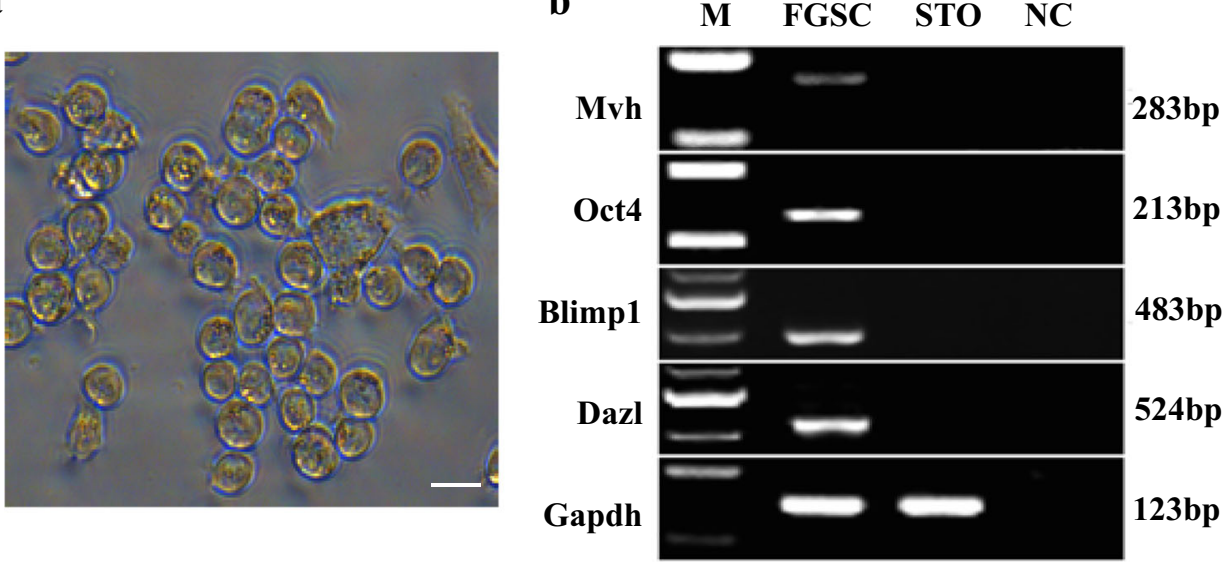

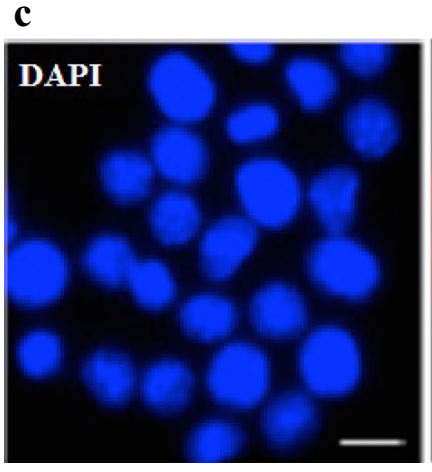

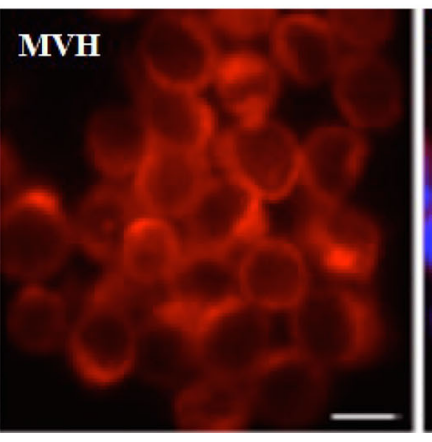

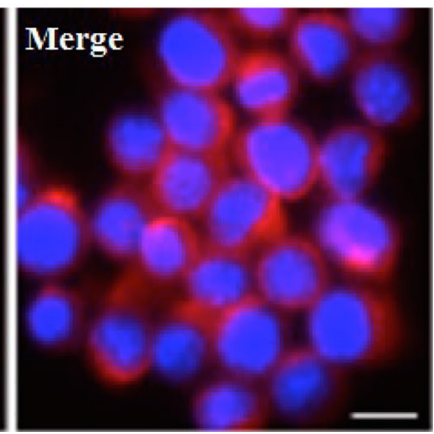

Usp7. The overexpression of Usp 7 was confirmed by qRT-PCR and western blotting. This overexpression led to $>6$-fold and $>$ 2.5-fold increases in mRNA (Fig. 4b) and protein levels (Fig. 4c) in FGSCs compared with those in control cells. The results showed that the overexpression of Usp 7 in FGSCs significantly inhibited their proliferation compared with that of controls (Fig. 4d, e). Accordingly, the expression of Oct4, Etv5, Foxol, and Akt was downregulated in Usp7-overexpressing FGSCs (Fig. 4f). Meanwhile, we found that marker genes related to differentiation, namely, Stra 8 and Sycp3, were upregulated in Usp7-overexpressing FGSCs compared with those in their control (Fig. 4g). Taking these findings together, the overexpression of Usp 7 inhibits FGSC growth and self-renewal and improves FGSC differentiation.

\section{Usp7 Regulates Female Germline Stem Cell Self- Renewal and Differentiation as Revealed by RNA-Seq}

To elucidate the mechanisms underlying Usp7's regulation of the self-renewal and differentiation of FGSCs, we performed RNAseq of FGSCs with Usp 7 knockdown and overexpression. FastQC was carried out to ensure the data quality (Fig. S1a-S1d). For each replicate, a Q-score higher than 30 (error $<0.1 \%$ ) was considered to suggest that the sequencing results were reliable. Overall, a total of 1044.2 million reads were generated. After applying a stringent filtering approach, 3802 differentially expressed genes were detected (adjusted $p$ value $<0.05$; fold change $>2$ ). There were 414 upregulated and 232 downregulated genes in Usp7-overexpressing FGSCs compared with the levels in their control, while there were 1446 upregulated and 1710 downregulated genes in the Usp7-knockdown FGSCs compared with the levels in their control. The differentially expressed mRNAs were calculated by MeV_4_9_0 cluster analysis to generate heat maps (Fig. 5a, b) and volcano plots (Fig. S2a, S2b). Some genes were also selected at random to verify the results of RNA-seq by qRT-PCR (Fig. S2c).

The differentially expressed genes were further analyzed with the Database for Annotation, Visualization and Integrated Discovery (DAVID; http://david.abcc.ncifcrf.gov) $[13,27]$. Gene Ontology (GO) and KEGG pathway analyses were used to determine the biological process terms particularly associated with the differentially expressed genes. The GO and KEGG pathway terms are shown in Fig. 5c-5f. GO terms in Usp7-knockdown FGSCs and their control showed particular associations with the cell cycle, regulation of cell proliferation, and cell division. Meanwhile, cell differentiation and fertilization were particularly associated with the Usp7-overexpressing FGSCs and their control. Moreover, DNA methylation and histone H3-K27 trimethylation were particularly identified, which reveals that Usp 7 may modulate FGSCs via DNA methylation or histone modification.

Furthermore, KEGG analysis terms in the Usp7-knockdown FGSCs and their control showed particular associations with the PI3K-Akt signaling pathway, Foxo signaling pathway, and 
Fig. 2 Usp7 expression is upregulated in differentiated female germline stem cells. $(\mathbf{a}, \mathbf{b})$ Relative expression of $U s p 7$ mRNA (a) and protein (b) in the ovary of postnatal mice at various times. (c) Microscopic observation of the morphological characteristics of in vitro differentiated FGSCs. (d) RTPCR identification of Sycp 3 and Stra8 in FGSCs differentiated in vitro. (e, f) Relative expression of $U s p 7$ mRNA (e) and protein (f) in FGSCs differentiated in vitro.

Values are mean $\pm \mathrm{SD}(\mathrm{n}=3)$.

$* P<0.05, * * P<0.01, * * * P<$ $0.001 .3 d 3$ days, $7 d 7$ days, $21 d$ 21 days, $42 d 42$ days. Dif in vitro differentiated FGSCs, con: FGSCs a

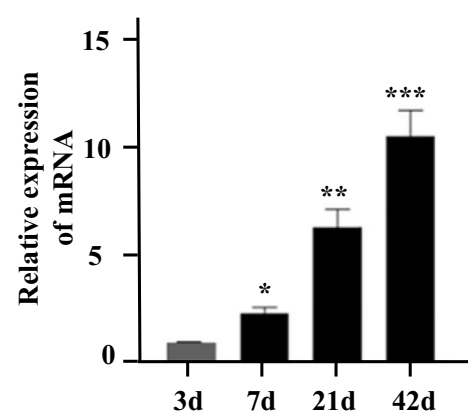

c

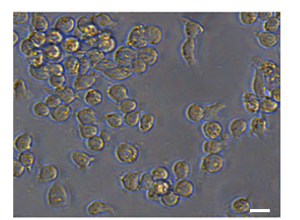

e

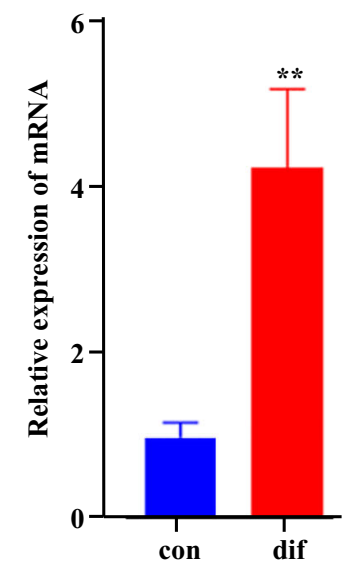

b
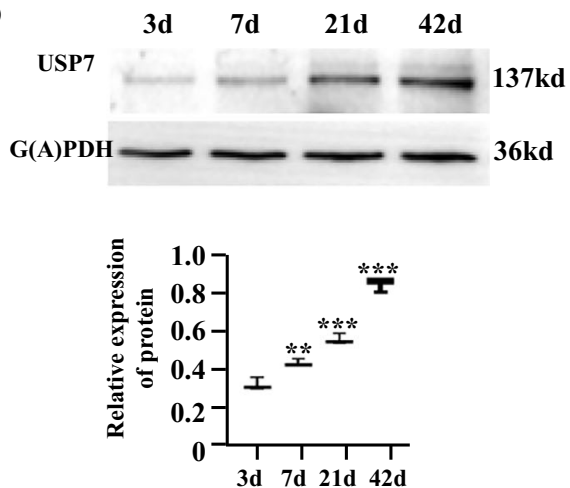

d

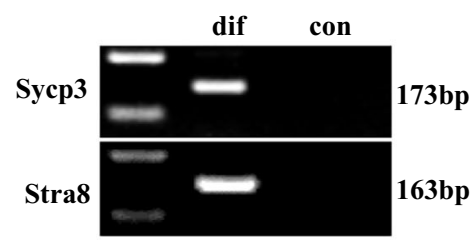

f

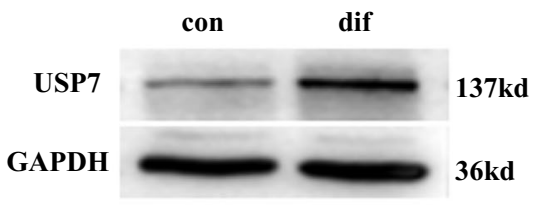

cGMP-PKG signaling pathway. Meanwhile KEGG analysis terms in the Usp7-overexpressing FGSCs and their control showed particular associations with protein digestion and absorption, p53 signaling pathway, and other pathways. These biological processes and pathways were consistent with our CCK8, EdU, and qRT-PCR results. In particular, the PI3K-Akt signaling pathway was confirmed to regulate FGSCs [19, 27], which meant that $U s p 7$ may modulate FGSCs via the PI3K-Akt signaling pathway.

\section{Usp7 Affects Female Germline Stem Cell Development through DNA Methylation}

To determine whether $U_{s p} 7$ modulates FGSCs via DNA methylation, dot blotting was performed (Fig. 6a). The results showed that DNA methylation is positively correlated with $U s p 7$ in FGSCs. The mRNA expression of Dnmt1, Dnmt3a, and Dnmt3b, known as methyltransferase genes [47], was analyzed using qRT-PCR (Fig. 6b). However, the mRNA expression of these DNA methyltransferases was increased in Usp7-knockdown FGSCs but showed no significant changes in Usp 7-overexpressing FGSCs. We deduced that Usp7 deubiquitinated these target proteins and protected them from degradation, which thus led to compensatory increases in the mRNA levels.

Furthermore, we determined the genomic DNA methylation with methylated DNA immunoprecipitation sequence (MeDIP-seq) in Usp 7-knockdown FGSCs and their control. Fast-QC was carried out to ensure the data quality (Fig. S3a$\mathrm{S} 3 \mathrm{~b})$. A total of 315.2 million reads yielding 38.1 million peaks were generated. Overall, 2059 genes were screened and showed different DNA methylation patterns. These 2059 genes were subjected to GO and KEGG pathway analyses. The GO terms are shown in Fig. 6c and KEGG terms in Fig. S4a. The GO terms showed particular associations with cell differentiation, regulation of cell proliferation, regulation 
Fig. 3 Knockdown of $U s p 7$ enhances female germline stem cell growth and self-renewal. (a) Fluorescence and bright field image of FGSCs infected with lentivirus. (b) qRT-PCR examined the expression level of Usp7 in FGSCs infected with Usp7-knockdown lentivirus or Usp 7-knockdown lentivirus control. (c) Top: Western blot analyses of the levels of USP7 in FGSCs infected with lentivirus. Bottom: Statistical results of western blotting. (d) CCK-8 assays were performed using FGSCs infected with Usp 7-knockdown lentivirus. (e) EdU assays were performed using FGSCs infected with Usp7knockdown lentivirus. (f) The expression of Oct4, Etv5, Foxo1, and $A k t$ in Usp7-knockdown FGSCs. (g) The expression of Strat8 and Sycp 3 in Usp7-knockdown FGSCs. Scale bars: $10 \mu \mathrm{m}$ in (a); $20 \mu \mathrm{m}$ in (e). $* P<0.05$;** $P<0.01$; $* * * P<0.001 . \mathrm{kd}-\mathrm{con}:$ Usp 7 knockdown control, kd: Usp7 knockdown

a
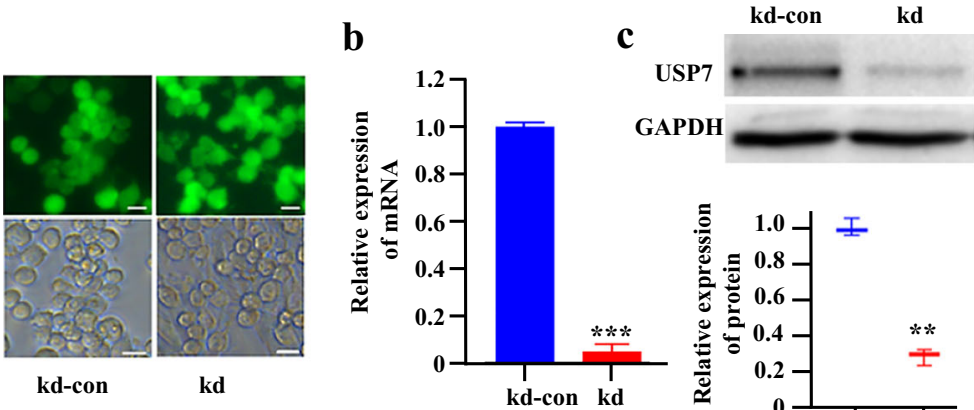

$137 \mathrm{kd}$

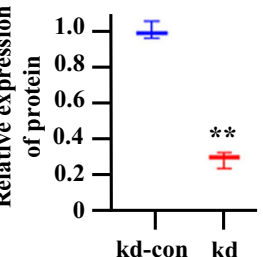

d

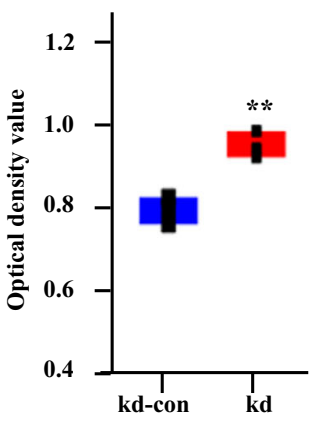

e
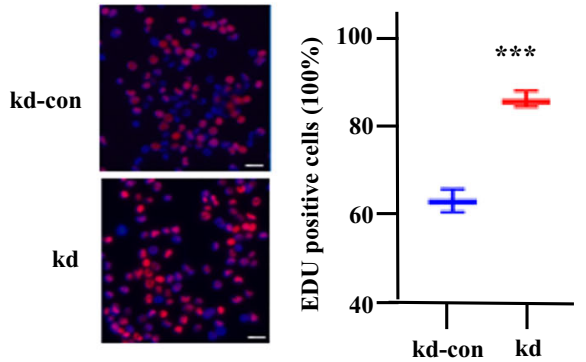

f

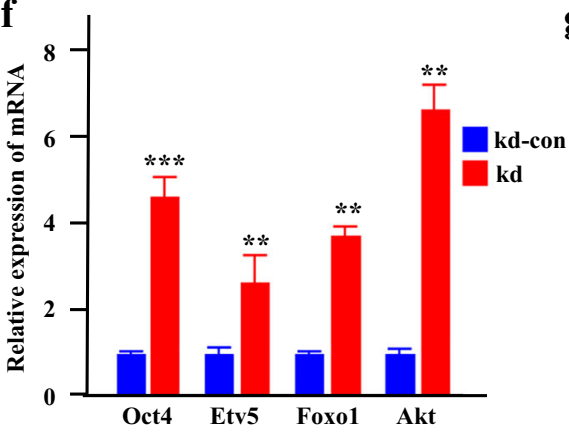

g

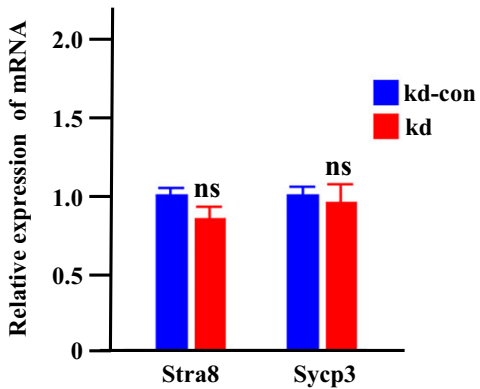

of cell growth, and negative regulation of cell growth. The KEGG pathway terms showed particular associations with the PI3K-Akt signaling pathway, MAPK signaling pathway, and Wnt signaling pathway. These GO and KEGG terms from the DNA methylation data reflected those from the RNA-seq analysis.

Additionally, upon comparing these 2059 genes in genomic DNA methylation and differentially expressed genes in RNAseq, we identified 255 overlapping genes. GO analysis was carried out again to further reveal the functions of DNA methylation. This showed that cell differentiation, regulation of cell proliferation, and regulation of gene expression were particularly associated with the GO terms (Fig. S4b). These results suggested that changes of DNA methylation may result in changes in gene expression, followed by the modulation of FGSCs. Thus, we speculated that Usp 7 may regulate the FGSCs by genomic methylation.

To determine how Usp 7 regulates the FGSCs by genomic methylation, decitabine, a DNA methyltransferase inhibitor, was used to identify whether inhibitor treatment of
Usp7-overexpressing FGSCs would rescue cell defects. Dot blotting confirmed that the level of genomic methylation was improved in Usp7-overexpressing FGSCs. Therefore, the Usp7-overexpressing FGSCs were treated with decitabine for $48 \mathrm{~h}$. Decitabine treatment transiently decreased the expression level of Dnmt1, Dnmt3a, and Dnmt3b as detected by RT-qPCR, indicating that the inhibitors worked as expected (Fig. S5). After treatment, the CCK 8 assay results showed that the viability value was increased from 0.524 to 0.695 , which was decreased from 0.776 to 0.524 by Usp7 overexpression in FGSCs (Fig. 6d). Meanwhile, qRT-PCR results showed that the gene expression of Etv5, Foxol, and Akt was improved at the mRNA level after treatment with decitabine (Fig. 6e). These observations suggested that inhibiting the process of DNA methylation rescued the growth defects of $U s p 7$-overexpressing FGSCs. Thus, we deduced that $U s p 7$ regulates FGSCs via the regulation of genomic DNA methylation. These results suggest that Usp 7 regulates FGSC proliferation and differentiation through DNA methylation. 
Fig. 4 Usp7 overexpression inhibits female germline stem cell growth and self-renewal, while improving FGSC differentiation. (a) Fluorescence and bright field image for FGSCs infected with lentivirus. (b) qRT-PCR examined the expression level of $U s p 7$ in FGSCs infected with

Usp7-overexpressing lentivirus or Usp 7-overexpressing lentivirus control. (c) Top: Western blot analyses of the levels of USP7 in FGSCs infected with lentivirus. Bottom: Statistical results of western blotting. (d) CCK-8 assays were performed using FGSCs infected with

Usp 7-overexpressing lentivirus.

(e) EdU assays were performed using FGSCs infected with Usp 7-overexpressing lentivirus.

(f) The expression of Oct4, Etv5, Foxol, and Akt in

Usp7-overexpressing FGSCs. (g)

The expression of strat 8 and sycp3 in Usp7-overexpressing FGSCs. Scale bars: $10 \mu \mathrm{m}$ in (a); $20 \mu \mathrm{m}$ in (e). $* \mathrm{P}<0.05$;** $\mathrm{P}<$ $0.01 ; * * * \mathrm{P}<0.001$. over-con: Usp 7 overexpression control, over: $U s p 7$ overexpression $\mathbf{a}$

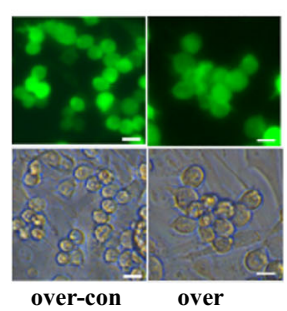

b

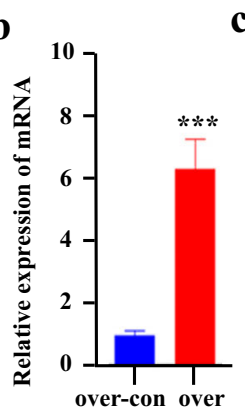

c

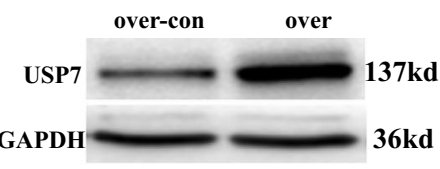

d

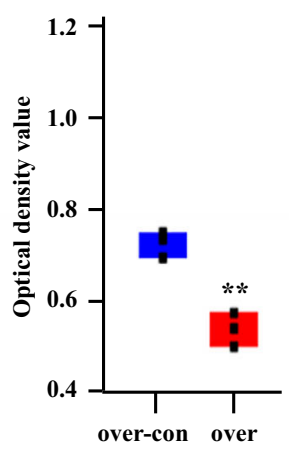

e
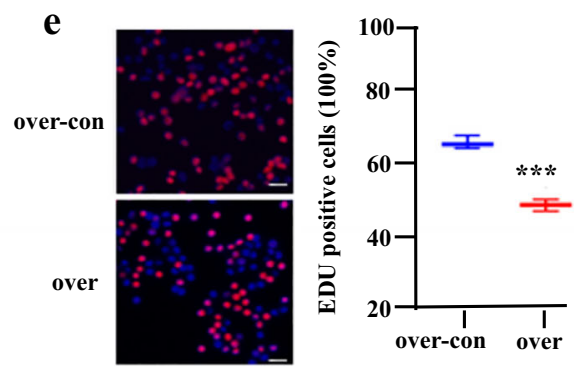

f

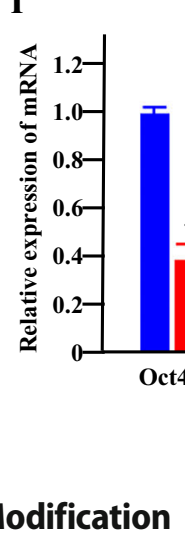

\section{Usp7 Affects Female Germline Stem Cell Development, but Not through Histone Modification}

To determine whether $U s p 7$ modulates FGSCs through histone modification, western blotting was used to determine the changes of $\mathrm{H} 3 \mathrm{~K} 27 \mathrm{me} 3$ and $\mathrm{H} 3 \mathrm{~K} 27 \mathrm{ac}$, and confirmed that $U s p 7$ positively regulates the level of $\mathrm{H} 3 \mathrm{~K} 27 \mathrm{me} 3$ (Fig. 7a), but negatively controls H3K27ac (Fig. 7b). We also tested the effect of $U s p 7$ on $\mathrm{H} 3 \mathrm{~K} 9 \mathrm{me} 3$, and the results showed that $U s p 7$ did not regulated the level of H3K9me3 (Fig. S6). We then performed H3K27me3 and H3K27ac ChIP-seq analysis in Usp7-knockdown FGSCs and their control. Fast-QC was carried out to ensure the data quality (Fig. S7a-S7f). A total of 1065.1 million reads were generated. Overall, 1563 and 1151 genes showed different $\mathrm{H} 3 \mathrm{~K} 27 \mathrm{me} 3$ and $\mathrm{H} 3 \mathrm{~K} 27 \mathrm{ac}$ modification patterns in Usp 7-knockdown FGSCs compared with their control. These 1563 genes from H3K27me3 ChIP-seq were subjected to GO and KEGG pathway analyses. The GO terms are shown in Fig. $7 \mathrm{c}$ and KEGG terms in Fig. S6a. The GO analysis suggested particular associations with the regulation of MAP kinase activity, fibroblast growth factor receptor signaling pathway, and regulation of cGMP metabolic process. KEGG analysis suggested particular associations with the Wnt signaling pathway, thyroid hormone signaling pathway, and chemical carcinogenesis. These 1151 genes from H3K27ac ChIP-seq were subjected to GO and KEGG pathway analyses. The GO terms are shown in Fig. 7d and KEGG terms in Fig. S6b. The GO analysis suggested particular associations with the regulation of gene expression, activation of MAPK activity, and Wnt signaling pathway. The KEGG analysis suggested particular associations with the MAPK signaling pathway, Ras signaling pathway, and cAMP signaling pathway.

Additionally, upon comparing these 1563 genes in the H3K27me3 ChIP-seq data and the differentially expressed genes in RNA-seq, we identified 213 overlapping genes. The $\mathrm{GO}$ analysis terms showed particular associations with the regulation of peptidyl-tyrosine phosphorylation, regulation of MAP kinase activity, and regulation of catalytic activity (Fig. S8c). Meanwhile, 
Fig. 5 RNA-seq reveals that Usp 7 alters female germline stem cell renewal and differentiation. (a, b) Hierarchical clustering shows differentially expressed mRNA patterns between Usp 7-knockdown FGSCs (a) and Usp7-overexpressing FGSCs (b) compared with the corresponding controls. (c, d) GO analysis of differentially expressed genes in Usp 7-knockdown (c) and overexpressing FGSCs (d) compared with the corresponding controls. GO analysis was performed by DAVID.

Differentially expressed genes had an adjusted $P<0.01$ and a twofold or greater difference in expression. (e, f) KEGG pathway terms of differentially expressed genes in Usp7-knockdown (e) and -overexpressing FGSCs (f). KEGG pathway analysis was performed by DAVID $\mathbf{a}$

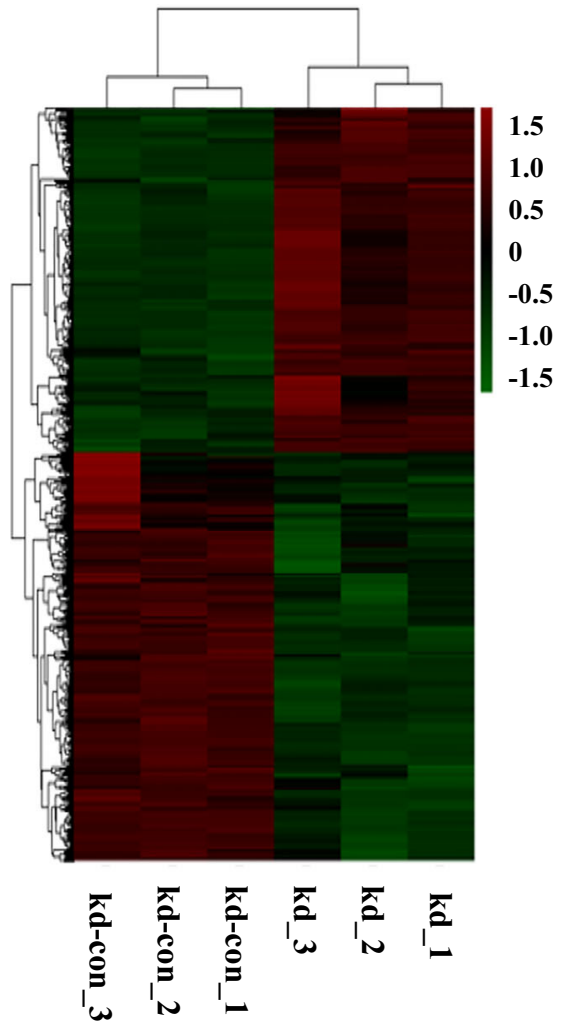

b
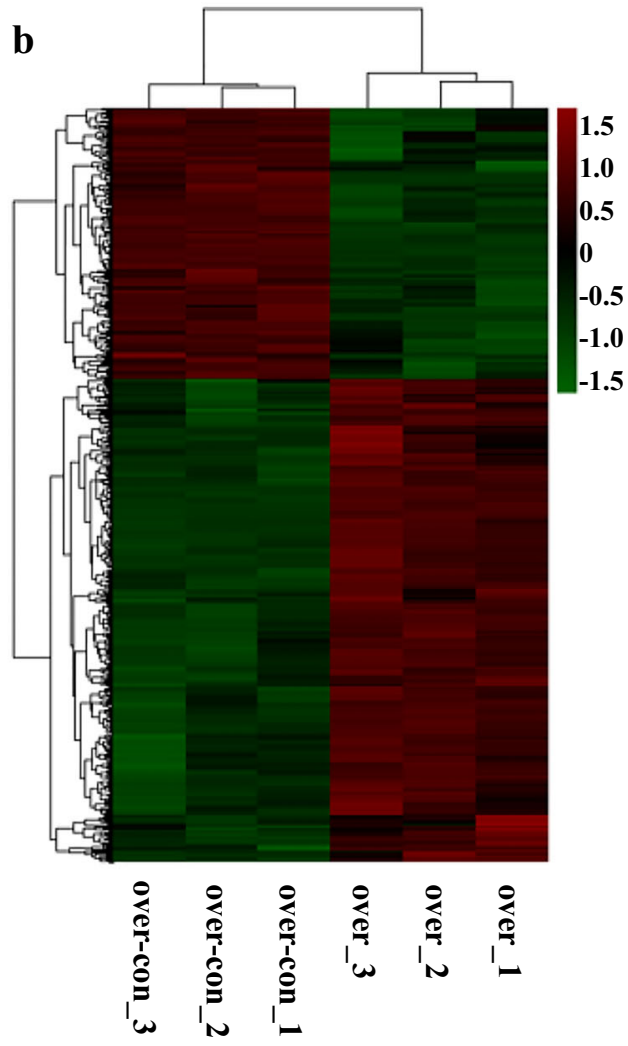

c

d

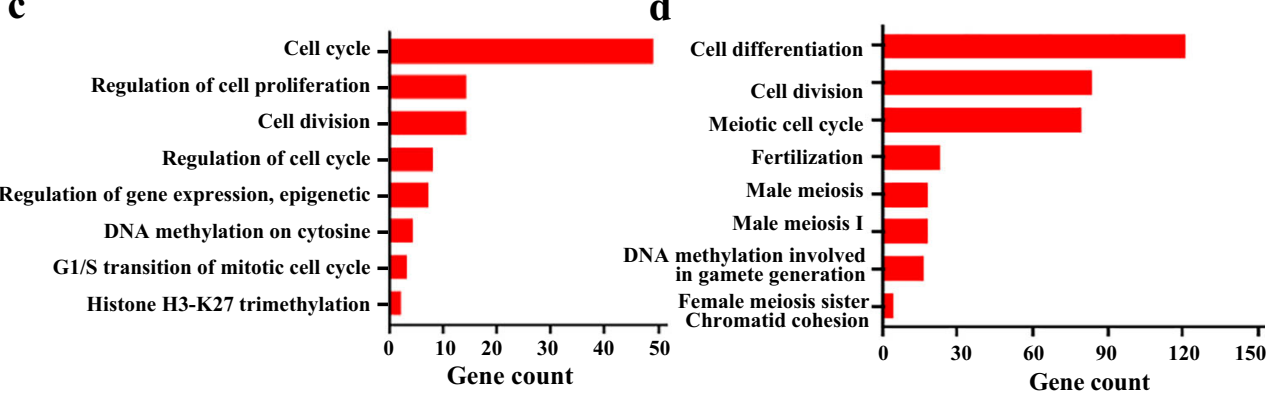

e

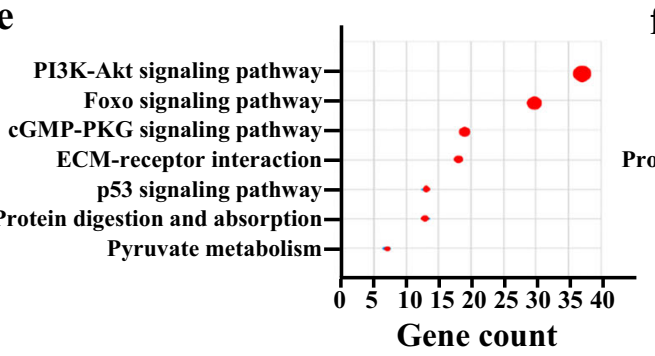

f

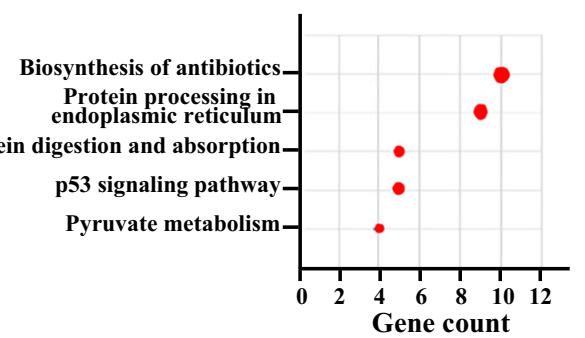

upon comparing these 1151 genes in the H3K27ac ChIP-seq data and the differentially expressed genes in RNA-seq, we identified 155 overlapping genes. The GO analysis results are shown in Fig. S6d. The GO analysis terms showed particular associations with nucleosome assembly, cell-cell adhesion, and regulation of cell death.

To identify the actual roles of H3K27me 3 and H3K27ac in FGSCs, histone methyltransferase inhibitor (GSK343), which represses EZH2-dependent H3K27me3 formation, and histone acetyltransferase inhibitor (C646), which restrains the activity of the $\mathrm{H} 3 \mathrm{~K} 27$ acetyltransferase crebbp (CBP)/p300, were used to treat the Usp7-overexpressing FGSCs and Usp 7-knockdown FGSCs to carry out a rescue assay. GSK343 treatment transiently decreased the expression level of Ezh2, and GSK343 treatment transiently decreased the expression level of $(\mathrm{CBP}) / \mathrm{p} 300$, indicating that the inhibitors worked as expected (Fig. S9). After the FGSCs had been treated with GSK343, CCK8 and qRT-PCR were performed again. The results showed that although Etv 5 and Akt were increased by GSK343 to some extent, the rescue effect was 
Fig. 6 Usp 7 affects female germline stem cell development through DNA methylation to modulate FGSCs. (a) Dot blotting was performed to identify the DNA methylation level in Usp 7-knockdown control, -knockdown, -overexpressing control, and -overexpressing FGSCs. (b) Relative expression of dnmt 1 , dnmt $3 a$, and dnmt $3 b$ at the mRNA level in Usp7knockdown control, -knockdown, -overexpressing control, and -overexpressing FGSCs. (c) GO analysis of the genes showing different DNA methylation patterns in Usp 7-knockdown FGSCs and their control. (d) CCK8 showing increased optical density value in Usp 7-overexpressing FGSCs treated with the DNA methylation inhibitor decitabine. (e) qRT-PCR showing the expression of Etv5, Foxol, and Akt genes in Usp 7-overexpressing FGSCs treated with the DNA methylation inhibitor decitabine. $* \mathrm{P}<0.05$, $* * \mathrm{P}<0.01, * * * \mathrm{P}<0.001 . n s$ no significant difference a

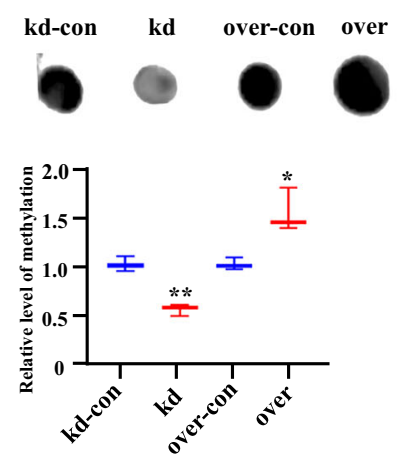

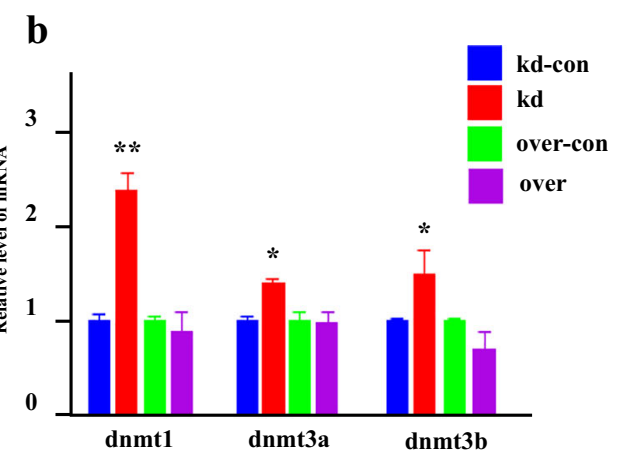

c

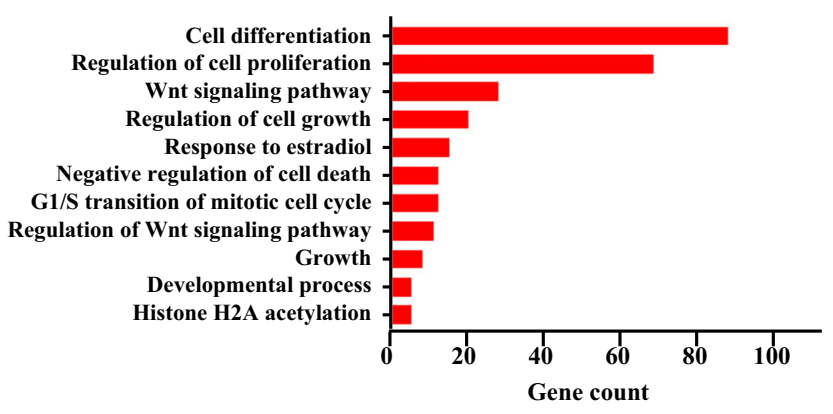

d

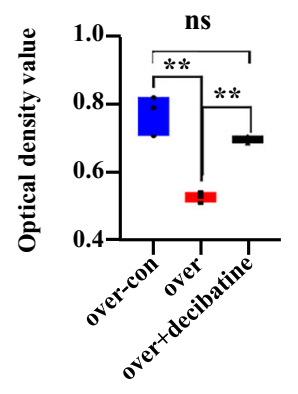

e

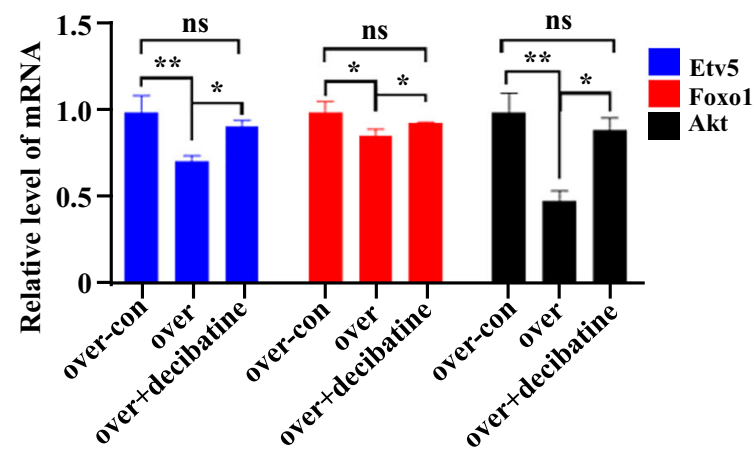

insufficient (Fig. 7e, f). Meanwhile, the results of CCK8 and qRT-PCR showed no changes after treatment with C646 (Fig. $7 \mathrm{~g}, \mathrm{~h})$. We deduced that $U s p 7$ regulates FGSCs at least in part through histone methylation, but this is not the key mechanism determining FGSC fate.

\section{Discussion}

Based on the above datas, we propose a model in which Usp7 alters DNA methylation by interacting with target proteins, such as DNMT1, DNMT3A, and DNMT3B, and modulates the DNA methylation level of genes related to the self-renewal and differentiation of FGSCs. This in turn results in a change in the fate determination of FGSCs.

In this study, we observed that the expression of Usp 7 was increased in ovary development by using qRT-PCR and western blotting on the ovaries of mice of different ages. The increased expression suggested that $U_{s p} 7$ may be involved in differentiation. It was identified by detecting differentiated FGSCs. The results are consistent with previous findings [36]. Meanwhile, accumulated research has shown that $U s p 7$ plays a vital role in cell development. Our results showed that $U s p 7$ overexpression negatively regulates FGSC self-renewal but positively modulates differentiation in FGSCs. This corresponds to the findings from the experiment in ovaries [Fig. 2a, b] and human adipose-derived stem cells [36]. Next, we performed RNA sequencing to elucidate the underlying mechanisms by which $U_{s p} 7$ regulates FGSC development. GO and KEGG analyses showed particular associations with the regulation of cell proliferation and cell division in Usp 7-knockdown FGSCs. Meanwhile, cell differentiation was particularly identified in the $U s p 7$-overexpressing FGSCs. RNAseq confirmed the findings of our previous assay. Additionally, given that DNA methylation was particularly identified among 
Fig. 7 Usp 7 does not regulate female germline stem cell development through histone modification. (a, b)

Representative western blots of acid-extracted histones from FGSCs using antibodies recognizing $\mathrm{H} 3 \mathrm{~K} 27 \mathrm{me} 3$ (a) and H3K27ac (b). The band sizes range from 17 to $23 \mathrm{kDa}$, as expected for modified histones. (c, d) GO analysis of the genes showing differential H3K27me3 (c) and $\mathrm{H} 3 \mathrm{~K} 27 \mathrm{ac}$ (d) modification patterns in Usp 7-knockdown FGSCs and their control. (e) Cell growth analysis based on a CCK8 assay of FGSCs treated with the H3K27me3 inhibitor GSK343.

The absorbance ratio of the overexpression group relative to controls is shown. (f) qRT-PCR showing the increased expression of Etv5, Foxo1, and Akt genes in Usp7-overexpressing versus control FGSCs treated with the H3K27me3 inhibitor GSK343.

(g) Cell growth analysis based on a CCK8 assay of FGSCs treated with the H3K27ac inhibitor C646. The absorbance ratio of the Usp7-knockdown group relative to controls is shown. (h) qRT-

PCR showing no obvious increase in the expression of Etv5, Foxol, and Akt genes in Usp7-knockdown versus control FGSCs treated with the H3K27ac inhibitor C646. $* \mathrm{P}<0.05$, **P $<$ $0.01, * * * \mathrm{P}<0.001 . n s$ no significant difference
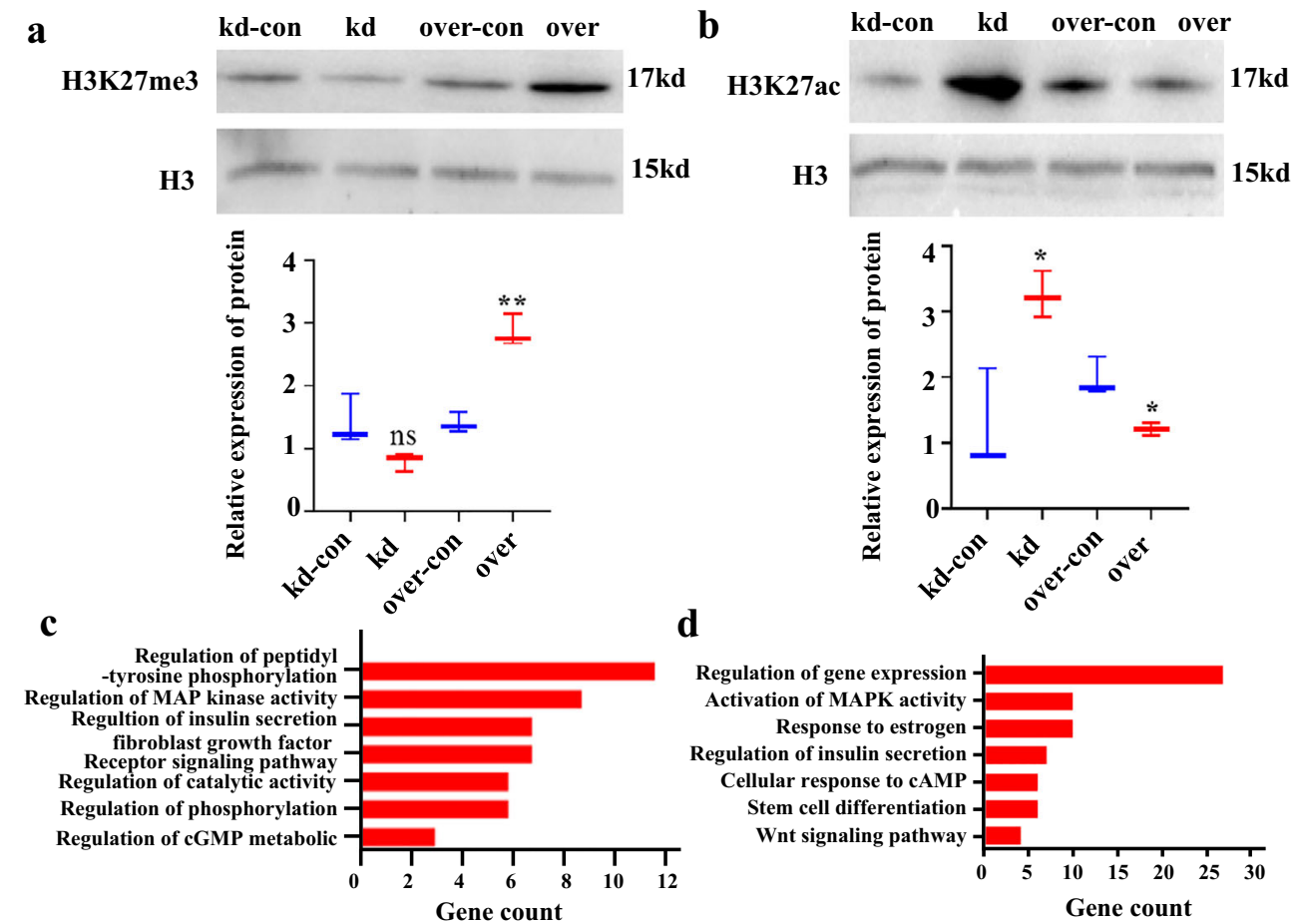

d
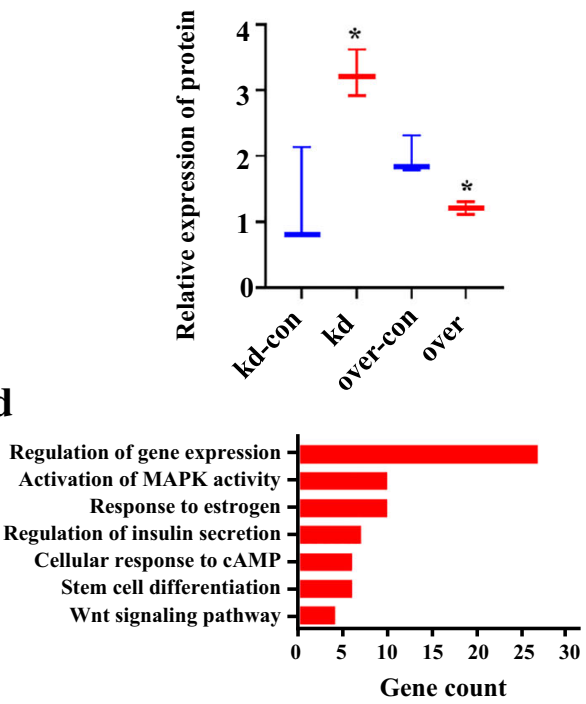

e

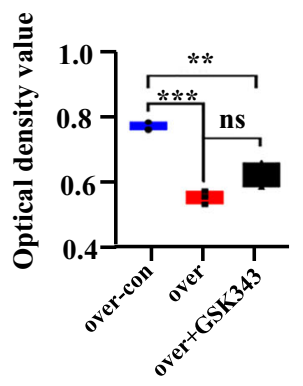

g

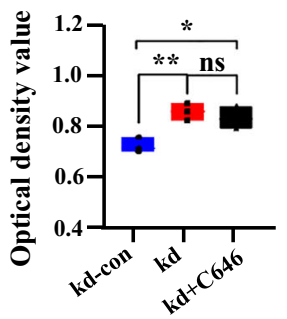

f

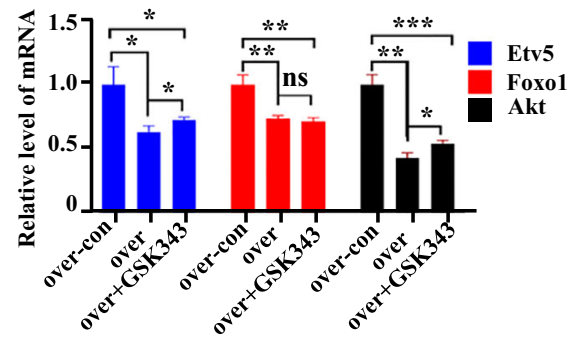

h

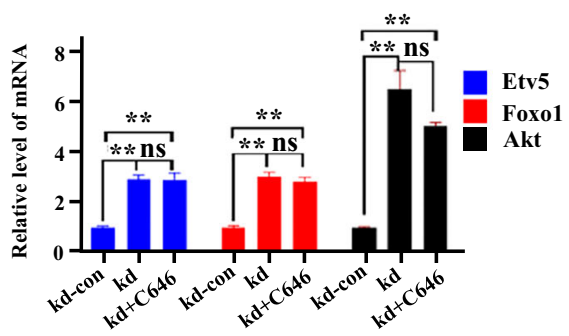

the GO terms and $U s p 7$ regulated the genomic methylation and modulated cell fate, a dot blot on FGSC DNA methylation was performed. This revealed that $U s p 7$ positively regulates DNA methylation in FGSCs. However, interestingly but confusingly, the mRNA expression of methyltransferases such as Dnmtl, $D n m t 3 a$, and Dnmt3b was upregulated in Usp7-knockdown FGSCs. However, there were hardly any changes in the Usp7-overexpressing FGSCs. We deduced that Usp 7 knockdown resulted in the increased degradation of these methyltransferases, which led to compensatory increases in mRNA levels. Based on the dot blot assay, MeDIP-seq was performed. The GO terms showed particular associations with cell differentiation and proliferation, which was consistent with the RNA-seq data and cell assay results. We deduced that Usp 7 regulates FGSCs through DNA methylation. To confirm this, decitabine, a DNA methyltransferase inhibitor, was used to carry out a rescue assay. The results of CCK8 and qRT-PCR suggested that decitabine rescued cell defects. This means that $U s p 7$ regulates FGSCs via DNA methylation. Moreover, H3K27 trimethylation was particularly identified in the RNA-seq GO analysis (Fig. 4d) and substantial research has confirmed that $U s p 7$ regulates cell fate through histone modification [10, 14, 17]. H3K27me3 is associated with gene silencing and $\mathrm{H} 3 \mathrm{~K} 27 \mathrm{ac}$ is associated with gene activation $[43,44]$. Thus, we performed western blotting to 
determine the relationship between $\mathrm{H} 3 \mathrm{~K} 27 \mathrm{me} 3$ or $\mathrm{H} 3 \mathrm{~K} 27 \mathrm{ac}$ levels and $U_{s p} 7$ expression in FGSCs. The western blotting results suggested that $U s p 7$ positively regulates the level of H3K27me3, but negatively controls that of H3K27ac. Therefore, we performed H3K27me3 and H3K27ac ChIP-seq analysis in Usp7-knockdown FGSCs and their control. The GO terms showed particular associations with the regulation of MAP kinase activity in the H3K27me3 ChIP-seq data. Meanwhile, gene expression, cell proliferation, activation of MAPK activity, and stem cell differentiation were particularly identified in the H3K27ac ChIP-seq data. Similarly, histone methyltransferase inhibitor (GSK343) and histone acetyltransferase inhibitor (C646) were each used to carry out rescue assays. qRT-PCR suggested that Etv 5 and Akt were partly upregulated by GSK343. However, the rescue effect was insufficient. Meanwhile, there were no obvious changes after treatment with C646. Additionally, upon comparing the GO terms in H3K27me3 or H3K27ac ChIP-seq and RNA-seq, there were still enormous changes. Thus, we deduced that $U s p 7$ regulates FGSCs though DNA methylation. The PI3K/Akt signaling pathway was enriched in KEGG terms in the RNA-seq and MeDIPseq data, but whether $U s p 7$ regulates FGSCs via the PI3K/Akt signaling pathway is unknown and needs further research.

The functional study of FGSCs is of great significance for our understanding of oogenesis and ovarian development. FGSCs were reported to have the capacity to differentiate into oocytes. Many women cannot produce normal eggs because of premature ovarian failure. Applying of FGSCs is a new strategy to solve the problem of female premature ovarian failure. However, there have been few reports about the molecular mechanisms of the proliferation and differentiation of FGSCs. Even less research has been performed about the regulation of FGSCs for an epigenetic perspective. However, epigenetic modification is of great significance in the process of gamete production and embryonic development. In this study, we found that $U s p 7$ regulates the proliferation and differentiation via DNA methylation.

It has been reported that deubiquitylases (DUBs) act to remove ubiquitin from their protein substrates and protect them from degradation [11]. Usp 7 is a deubiquitylase that is classified into the ubiquitin-specific protease (USP) family. Usp 7 targets various proteins, such as chromatin-associated factors (DNMT1 [8], and UHRF1 [48]), as well as tumor suppressors (p53 [5], PTEN [35]). It is well established that Usp 7 plays diverse roles in genome stability, cell cycle, apoptosis, proliferation, and differentiation. An assay on the knockout of Usp 7 in mice showed that embryonic growth stagnated and death occurred between E6.5 and E7.5 [16]. USP7 associates with KDF1 and regulates skin differentiation through the deubiquitination and stabilization of IKK $\alpha$ [21]. Generating mice with conditional knockout in the ovaries would be a meaningful approach to further define the functions of $U s p 7$ in mouse ovarian development.
DNA methylation, in which a methyl group is added to carbon 5 of the cytosine in the $\mathrm{CpG}$ context, is an essential epigenetic modification. It has been reported to play a vital role in many biological processes, such as the regulation of gene expression [12], X-chromosome inactivation [3], genetic imprinting [33], and disease formation [15]. In particular, the level of genomic DNA methylation was found to be changed in the process of self-renewal and differentiation of stem cells. In germ cells, DNA methylation can be inherited through cell division and transmitted from one generation to the next. Therefore, clarification of the DNA methylation of FGSCs is vital to understand oogenesis. Our study revealed that $U_{s p} 7$ overexpression induces FGSC differentiation. We deduced that $U s p 7$ may affect gamete production and embryonic development. However, more work in this field of study is needed.

To the best of our knowledge, our study is the first to evaluate the effects of $U s p 7$ in FGSCs in vitro, which suggests that $U_{s p} 7$ is essential for the fate determination of FGSCs by DNA methylation. This study provides novel mechanisms that control FGSC fate and enables deep insights for understanding FGSC development.

\section{Materials and Methods}

\section{Culture of Female Germline Stem Cells}

The FGSCs were cultured as described previously [42, 49, 50]. In brief, FGSCs were cultured on mitomycin C-treated $(10 \mu \mathrm{g} / \mathrm{ml}$, Sigma) mitotically inactivated mouse STO (derived from mouse SIM embryonic fibroblasts, strain SIM, $5 \times 10^{4}$ cells $/ \mathrm{cm}^{2}$, ATCC) cell feeders. The culture medium for FGSCs was Minimum Essential Medium-alpha (MEMalpha; Invitrogen, Carlsbad, CA, USA), supplemented with $10 \%$ fetal bovine serum (FBS), $10 \mathrm{ng} / \mathrm{ml}$ mouse leukemia inhibitory factor (mLIF; Santa Cruz Biotechnology, CA, USA), $10 \mathrm{ng} / \mathrm{ml}$ mouse basic fibroblast growth factor (mbFGF; BD Biosciences, Franklin Lakes, NJ, USA), $10 \mathrm{ng} / \mathrm{ml}$ mouse epidermal growth factor (mEGF; PeproTech, NJ, USA), $40 \mathrm{ng} / \mathrm{ml}$ mouse glial cell linederived neurotrophic factor (mGDNF; R\&D Systems, Minneapolis, MN, USA), $1 \mathrm{mM}$ non-essential amino acids (Invitrogen Life Sciences, MA, USA), 2 mM L-glutamine (Amresco, Lardner, PA, USA), $10 \mathrm{mg} / \mathrm{ml}$ penicillin (Amresco), $30 \mathrm{mg} / \mathrm{ml}$ pyruvate (Amresco), and $\beta$ mercaptoethanol (Sigma-Aldrich, St. Louis, MO, USA). The FGSCs were sub-cultured every 4-6 days at a 1:3 ratio.

\section{Lentiviral Packaging}

For Usp7-knockdown lentiviral vectors, we purchased lentivirus packaging plasmids and pGMLV-SC5 lentiviral vectors 
from Genomeditech Biotechnology Co., Ltd. (Shanghai, China). A puromycin selection site was included for selecting cells in the vector plasmid. We designed a sequence, GCCGAATTAACAGAGAGAAT, as a shRNA target site for Usp 7 knockdown. The blank lentiviral vector was used as the Usp 7-knockdown control.

For Usp 7-overexpressing lentiviral vectors, we purchased lentivirus packaging plasmids and pRLenti-CMV-MCS-HA3Flag-P2A-EGFP lentiviral vectors. We designed a primer sequence for $U s p 7$ overexpression Primer (F): AAGCTTGT GACGTCTCGGT, Primer (R): GGATCCCCAAAGTT CTAGGC. The blank lentiviral vector was used as the Usp 7-overexpressing control.

Inhibition or overexpression plasmids and lentivirus packaging plasmids were co-transfected into HEK293T cells to generate lentivirus particles. At $12 \mathrm{~h}$ after transfection, enhancing buffer was added. At $48 \mathrm{~h}$ after transfection, virus particles were collected by centrifugation.

\section{Lentiviral Infection}

The FGSCs were normally cultured to a confluence of $40 \%$ on a 48-well plate and incubated with a 1:1 mixture of culture medium and lentivirus concentrated solution (lentivirus titer: $\left.1 \times 10^{9} \mathrm{TU} / \mathrm{ml}\right)$, supplemented with $5 \mu \mathrm{g} / \mathrm{ml}$ polybrene. After $12 \mathrm{~h}$ of infection, the cells were cultured with normal FGSC medium for $24 \mathrm{~h}$. Then, they were infected with lentiviral particles and screened with puromycin to obtain Usp7-overexpressing or -knockdown FGSCs.

\section{CCK8 Assay}

Lentivirus-infected cells were seeded on a 96-well plate, with 5000 cells per well and $200 \mu \mathrm{l}$ of culture medium. When the cell density reached $70-80 \%$, CCK 8 reagent was added to the plate $(20 \mu \mathrm{l} / \mathrm{well})$, followed by incubation for $2 \mathrm{~h}$ at $37^{\circ} \mathrm{C}$ and $5 \% \mathrm{CO}_{2}$. The light absorption value was measured at $450 \mathrm{~nm}$ using a microplate reader (Bio-Tek Instruments, Thermo Fisher Scientific, Winooski, VT, USA).

\section{EdU Assay}

The cells were normally cultured to a density of $80 \%$ and incubated with $50 \mu \mathrm{M}$ EdU reagent for $2 \mathrm{~h}$. Then, $4 \%$ paraformaldehyde(PFA)was used to fix the cells at room temperature for $30 \mathrm{~min}$, followed by neutralization with $2 \mathrm{mg} / \mathrm{ml}$ glycine for $5 \mathrm{~min}$. Next, $0.5 \%$ Triton $\mathrm{X}-100$ was used to treat the cells. The cells were supplemented with $1 \times$ Apollo staining solution and incubated for $30 \mathrm{~min}$, followed by washing with PBS containing $0.5 \%$ Triton X-100 three times. Then, $1 \times$ Hoechst 33342 was used to dye the cell nuclei. Images were obtained with a Leica fluorescence microscope.

\section{Quantitative Real-Time Polymerase Chain Reaction}

The total RNA of FGSCs was extracted using Trizol reagent (QIAGEN, Hilden, Germany) and its quality was analyzed using Nanodrop Lite (Thermo Fisher Scientific, Winooski, VT, USA). A total of $1000 \mathrm{ng}$ of RNA was reversetranscribed into cDNA in a volume of $20 \mu \mathrm{l}$ using a reversetranscription kit (Takara, Tokyo, Japan). PCR was performed with Taq DNA polymerase. Quantitative real-time polymerase chain reaction (qRT-PCR) analysis was carried out with SYBR Premix E $\times$ Taq (Takara, Shanghai, China) using an Applied Biosystems $® 7500$ Real-Time PCR System. The data were analyzed by the $2^{-\Delta \Delta \mathrm{Ct}}$ method. The primers are shown in Table S1.

\section{Immunofluorescence Staining}

Cells were cultured in a 48 -well plate to $80 \%$ density. Then, they were washed twice with phosphate buffer saline (PBS) and fixed with $4 \%$ PFA for $30 \mathrm{~min}$ at room temperature. The cells were blocked with $10 \%$ goat serum at $37{ }^{\circ} \mathrm{C}$ for $30 \mathrm{~min}$. After washing twice with PBS, the cells were incubated with an anti-MVH antibody in PBS (1:100; Abcam, Cambridge, MA, USA) overnight at $4{ }^{\circ} \mathrm{C}$. The cells were incubated with tetramethylrhodamine isothiocyanate (TRITC)-conjugated secondary antibody in PBS at $37^{\circ} \mathrm{C}$ for $1 \mathrm{~h}$ (goat anti-rabbit $\mathrm{IgG}$; ProteinTech). Then, the cells were incubated at $37^{\circ} \mathrm{C}$ for $5 \mathrm{~min}$ with $500 \mathrm{ng} / \mathrm{ml}$ 4', 6-diamidino-2-phenylindole (DAPI). Photographs were obtained using a Leica XP8 fluorescence confocal microscope (Leica, Wetzlar, Germany).

\section{Western Blotting}

The cells were cultured in 35-mm dishes to $90 \%$ density. They were then digested with $0.05 \%$ trypsin, washed twice with PBS, and lysed with $200 \mu \mathrm{l}$ of RIPA buffer (Yeasen, Shanghai, China) containing a protease inhibitor cocktail. Protein was obtained by centrifugation $\left(4{ }^{\circ} \mathrm{C}, 12,000 \times g, 10 \mathrm{~min}\right)$. Protein concentration was measured using a bicinchoninic acid (BCA) protein assay kit (Yeasen, Shanghai, China). After separation by $15 \%$ SDSPAGE gel electrophoresis, the proteins were transferred to polyvinylidene fluoride (PVDF) membranes. Each membrane was blocked with 5\% skim milk in Tris-buffered saline with Tween 20 (TBST) for $2 \mathrm{~h}$ with shaking at $37{ }^{\circ} \mathrm{C}$. Then, the membrane was incubated with the primary antibody (rabbit-anti-Usp7, 1:6000, Abcam; mouse-anti-gapdh, 1:8000, Abcam) at $4{ }^{\circ} \mathrm{C}$ overnight. The membrane was then washed three times with TBST for 10 min each time and incubated with the corresponding horseradish peroxidase-conjugated secondary antibody for $1 \mathrm{~h}$ at room temperature. Tanon 4600SF (Tanon, Shanghai, China) was used to scan protein bands. The grayscale value of the bands was calculated using ImageJ software. 


\section{RNA-Seq}

FGSCs were collected and processed with Trizol reagent (Life Technologies, CA, USA) to extract total RNA. Then, $1000 \mathrm{ng}$ of RNA was used to construct sequencing libraries using the VAHTSTM mRNA-seq v2 Library Prep kit for Illumina1 (Vazyme, Co., Ltd., Shanghai, China). In brief, random hexamer primers were used to synthesize first- and secondstrand cDNA, and the cDNA fragments were repaired with the End-It DNA End Repair kit. After adaptor ligation, an A nucleotide was added at the $3^{\prime}$ end of the fragments. The cDNA was amplified by PCR. The library quality was assessed using the Bio Analyzer 2100 (Agilent, Santa Clara, CA, USA). The Illumina HiSeq 2500 platform (Illumina, San Diego, CA, USA) was applied for RNA-seq, and FastQC was used to evaluate the quality of RNA Sequencing (RNA-seq) data.

\section{Dot Blotting}

Genomic DNA was extracted using TIANamp Genomic DNA Kit. Diluted DNA (50 ng/ $\mu \mathrm{l}$ ) was blotted to an activated PVDF membrane. After cross-linking with a UV cross-linker, the membrane was blocked with $5 \%$ skim milk and then incubated with an antibody to $5 \mathrm{mc}(\mathrm{ab} 10805,1: 500)$ at $4{ }^{\circ} \mathrm{C}$ overnight. After washing three times with TBST for $10 \mathrm{~min}$, the membrane was incubated with the secondary antibody for $1 \mathrm{~h}$ at room temperature and then the dots were scanned with Tanon 4600SF (Tanon, Shanghai, China).

\section{Methylated DNA Immunoprecipitation Sequence}

The work of preparing the MeDIP and DNA libraries was carried out as described previously [26]. In brief, we extracted genomic DNA and cleared the redundant RNA with RNase. Then, the genomic DNA was broken into fragments of about $300 \mathrm{bp}$. The fragmented chromatin was immunoprecipitated with protein $A+G$ magnetic beads bound with 5-methylcytosine antibody. Methylated DNA immunoprecipitation sequence (MeDIP-seq) and input DNA fragments were end-repaired and A-tailed applying the NEBNext End Repair/dA-Tailing Module (E7442, NEB), followed by adaptor ligation with the NEBNext Ultra Ligation Module (E7445, NEB). The DNA libraries were expanded, followed by deep sequencing with an Illumina HiSeq 2000 .

\section{Chromatin Immunoprecipitation Sequence}

The ChIP was carried out in accordance with a previously described protocol [39]. Briefly, cells were cross-linked using $1 \%$ formaldehyde, lysed using rotation, and broken using an ultrasonic breaker (Diagenode Bioruptor Pico, Belgium). MAGnify Chromatin Immunoprecipitation System (Thermo Fisher Scientific) was used to perform immunoprecipitation
(IP) as per the manufacturer's instructions with the antibody rabbit anti-H3K27ac (Abcam, Ab4729) or rabbit antiH3K27me3 (Millipore, 07-449). Chromatin samples were sent for high-throughput sequencing. We used Bowtie2 (v2.3.1) to align the chromatin immunoprecipitation sequence (ChIP-seq) DNA reads to the reference genome. Each ChIP library was compared against the DNA input background library in the corresponding cell type condition. Specifically, histone marks H3K27me3 and H3K27ac in Usp 7 FGSCs and Usp7-knockdown FGSC control were compared with the DNA input Usp7-knockdown FGSCs and Usp7-knockdown FGSC control, respectively. FDR $<0.05$ was used to determine ChIP-seq peaks relative to the input libraries.

\section{Gene Ontology and Kyoto Encyclopedia of Gene and Genomic Pathway Analysis}

The biochemical processes involving the differentially expressed mRNAs and genes showing different DNA methylation, H3K27me3, and H3K27ac patterns were elucidated by Gene Ontology (GO) analysis. The signal pathways of the mRNAs and genes were elucidated by Kyoto Encyclopedia of Gene and Genomic (KEGG) analysis. The data were uploaded to DAVID (http://david.abcc.ncifcrf.gov/home.jsp) and the enrichment results were obtained. Fisher's exact test was applied to evaluate the results, and the false discovery rate (FDR) was used to correct the $p$ values ( $\mathrm{p}$ value $<0.05$; fold change $>2$ ).

\section{Rescue Assay}

These inhibitors (C646, GSK343 and decitabine; MedChemExpress, Shanghai, China) were initially dissolved in $10 \mathrm{mM}$ DMSO to a range of concentrations (C646: 1, 2, 4, 6, 8, and $10 \mu \mathrm{M}$; GSK343: 1, 2, 4, 6, 8, and $10 \mu \mathrm{M}$; decitabine: 1, 2, $4,6,8$, and $10 \mu \mathrm{M}$ ) with FGSC medium. The FGSCs were cultured to a density of $40 \%$ on a 48 -well plate and treated with inhibitor for $24 \mathrm{~h}$. Finally, the appropriate concentrations of different inhibitors (C646: $4 \mu \mathrm{M}$; GSK343: $4 \mu \mathrm{M}$; decitabine: $8 \mu \mathrm{M})$ were determined according to the results of CCK8.

\section{Statistical Analysis}

All experiments in the study were replicated at least three times. The data are presented as mean \pm SEM. Student's $t$ test was performed with SPSS software. Differences were considered to be statistically significant at $p<0.05$.

Availability of Data and Material Raw sequencing reads were obtained from Gene Expression Omnibus and processed with the parameters detailed below: GSE155548

Code Availability (Software Application or Custom Code) Not applicable. 
Author Contributions YZ, XL, GT and XZ conducted all the major experiments, and data analysis. YZ and XL wrote the manuscript. JmW provided the usp 7 overexpression vector. YS performed RT-PCR to identify the female germline stem cells. JW initiated and supervised the entire project.

Funding This work was supported by National Basic Research Program of China (2018YFC1003501, 2017YFA0504201), National Nature Science Foundation of China (81720108017), the National Major Scientific Instruments and Equipment Development Project, National Nature Science Foundation of China (61827814).

\section{Compliance with Ethical Standards}

Conflict of Interest No competing interests declared.

Consent to Participate Not applicable.

\section{Consent for Publication Not applicable}

Open Access This article is licensed under a Creative Commons Attribution 4.0 International License, which permits use, sharing, adaptation, distribution and reproduction in any medium or format, as long as you give appropriate credit to the original author(s) and the source, provide a link to the Creative Commons licence, and indicate if changes were made. The images or other third party material in this article are included in the article's Creative Commons licence, unless indicated otherwise in a credit line to the material. If material is not included in the article's Creative Commons licence and your intended use is not permitted by statutory regulation or exceeds the permitted use, you will need to obtain permission directly from the copyright holder. To view a copy of this licence, visit http://creativecommons.org/licenses/by/4.0/.

\section{References}

1. Agathanggelou, A., Smith, E., Davies, N. J., Kwok, M., Zlatanou, A., Oldreive, C. E., Mao, J., Da Costa, D., Yadollahi, S., Perry, T., Kearns, P., Skowronska, A., Yates, E., Parry, H., Hillmen, P., Reverdy, C., Delansorne, R., Paneesha, S., Pratt, G., Moss, P., Taylor, A. M. R., Stewart, G. S., \& Stankovic, T. (2017). USP7 inhibition alters homologous recombination repair and targets CLL cells independently of ATM/p53 functional status. Blood, 130(2), 156-166.

2. Biswas, K., Philip, S., Yadav, A., Martin, B. K., Burkett, S., Singh, V., Babbar, A., North, S. L., Chang, S., \& Sharan, S. K. (2018). BRE/BRCC45 regulates CDC25A stability by recruiting USP7 in response to DNA damage. Nature Communications, 9(1), 537.

3. Cantone, I., \& Fisher, A. G. (2017). Human X chromosome inactivation and reactivation: Implications for cell reprogramming and disease. Philosophical Transactions of the Royal Society of London. Series B, Biological Sciences, 372(1733), 20160358.

4. Cheng, J., Yang, H., Fang, J., Ma, L., Gong, R., Wang, P., Li, Z., \& $\mathrm{Xu}, \mathrm{Y}$. (2015). Molecular mechanism for USP7-mediated DNMT1 stabilization by acetylation. Nature Communications, 6, 7023.

5. Cui, H., Guo, M., Xu, D., Ding, Z. C., Zhou, G., Ding, H. F., Zhang, J., Tang, Y., \& Yan, C. (2015). The stress-responsive gene ATF3 regulates the histone acetyltransferase Tip60. Nature Communications, 6, 6752.

6. Daigneault, B. W., Rajput, S., Smith, G. W., \& Ross, P. J. (2018). Embryonic POU5F1 is required for expanded bovine blastocyst formation. Scientific Reports, 8(1), 7753.
7. Ding, X., Liu, G., Xu, B., Wu, C., Hui, N., Ni, X., Wang, J., Du, M., Teng, X., \& Wu, J. (2016). Human GV oocytes generated by mitotically active germ cells obtained from follicular aspirates. Scientific Reports, 6, 28218.

8. Du, Z., Song, J., Wang, Y., Zhao, Y., Guda, K., Yang, S., Kao, H. Y., Xu, Y., Willis, J., Markowitz, S. D., Sedwick, D., Ewing, R. M., \& Wang, Z. (2010). DNMT1 stability is regulated by proteins coordinating deubiquitination and acetylation-driven ubiquitination. Science Signaling, 3(146), ra80.

9. Felle, M., Joppien, S., Nemeth, A., Diermeier, S., Thalhammer, V., Dobner, T., Kremmer, E., Kappler, R., \& Langst, G. (2011). The USP7/Dnmt1 complex stimulates the DNA methylation activity of Dnmt1 and regulates the stability of UHRF1. Nucleic Acids Research, 39(19), 8355-8365.

10. Gao, Y., Koppen, A., Rakhshandehroo, M., Tasdelen, I., van de Graaf, S. F., van Loosdregt, J., van Beekum, O., Hamers, N., van Leenen, D., Berkers, C. R., Berger, R., Holstege, F. C., Coffer, P. J., Brenkman, A. B., Ovaa, H., \& Kalkhoven, E. (2013). Early adipogenesis is regulated through USP7-mediated deubiquitination of the histone acetyltransferase TIP60. Nature Communications, 4, 2656.

11. Hanpude, P., Bhattacharya, S., Dey, A. K., \& Maiti, T. K. (2015). Deubiquitinating enzymes in cellular signaling and disease regulation. IUBMB Life, 67(7), 544-555.

12. Iida, T., Iwanami, A., Sanosaka, T., Kohyama, J., Miyoshi, H., Nagoshi, N., Kashiwagi, R., Toyama, Y., Matsumoto, M., Nakamura, M., \& Okano, H. (2017). Whole-genome DNA methylation analyses revealed epigenetic instability in tumorigenic human iPS cell-derived neural stem/ progenitor cells. Stem Cells, 35(5), 1316-1327.

13. Jia, W., Xu, B., \& Wu, J. (2018). Circular RNA expression profiles of mouse ovaries during postnatal development and the function of circular RNA epidermal growth factor receptor in granulosa cells. Metabolism, 85, 192-204.

14. Jiang, L., Xiong, J., Zhan, J., Yuan, F., Tang, M., Zhang, C., Cao, Z., Chen, Y., Lu, X., Li, Y., Wang, H., Wang, L., Wang, J., Zhu, W. G., \& Wang, H. (2017). Ubiquitin-specific peptidase 7 (USP7)mediated deubiquitination of the histone deacetylase SIRT7 regulates gluconeogenesis. The Journal of Biological Chemistry, 292(32), 13296-13311.

15. Kalish, J. M., Jiang, C., \& Bartolomei, M. S. (2014). Epigenetics and imprinting in human disease. The International Journal of Developmental Biology, 58(2-4), 291-298.

16. Kon, N., Kobayashi, Y., Li, M., Brooks, C. L., Ludwig, T., \& Gu, W. (2010). Inactivation of HAUSP in vivo modulates 553 function. Oncogene, 29(9), 1270-1279.

17. Lei, A., Chen, L., Zhang, M., Yang, X., Xu, L., Cao, N., Zhang, Z., \& Cao, Y. (2019). EZH2 regulates protein stability via recruiting USP7 to mediate neuronal gene expression in Cancer cells. Frontiers in Genetics, 10, 422.

18. Li, Y., Zhang, Z., Chen, J., Liu, W., Lai, W., Liu, B., Li, X., Liu, L., Xu, S., Dong, Q., Wang, M., Duan, X., Tan, J., Zheng, Y., Zhang, P., Fan, G., Wong, J., Xu, G. L., Wang, Z., Wang, H., Gao, S., \& Zhu, B. (2018). Stella safeguards the oocyte methylome by preventing de novo methylation mediated by DNMT1. Nature, 564(7734), 136-140.

19. Li, X., Hu, X., Tian, G. G., Cheng, P., Li, Z., Zhu, M., Zhou, H., \& Wu, J. (2019). C89 induces autophagy of female germline stem cells via inhibition of the PI3K-Akt pathway in vitro. Cells, 8(6), 606.

20. Li, X., Tian, G. G., Zhao, Y., \& Wu, J. (2019). Genome-wide identification and characterization of long noncoding and circular RNAs in germline stem cells. Science Data, 6(1), 8.

21. Li, Y., Tang, L., Yue, J., Gou, X., Lin, A., Weatherbee, S. D., \& $\mathrm{Wu}, \mathrm{X}$. (2020). Regulation of epidermal differentiation through KDF1-mediated deubiquitination of IKKalpha. EMBO Reports, 21(5), e48566.

22. Liang, L., Peng, Y., Zhang, J., Zhang, Y., Roy, M., Han, X., Xiao, X., Sun, S., Liu, H., Nie, L., Kuang, Y., Zhu, Z., Deng, J., Xia, Y., 
Sankaran, V. G., Hillyer, C. D., Mohandas, N., Ye, M., An, X., \& Liu, J. (2019). Deubiquitylase USP7 regulates human terminal erythroid differentiation by stabilizing GATA1. Haematologica, 104(11), 2178-2187.

23. Liefke, R., Karwacki-Neisius, V., \& Shi, Y. (2016). EPOP interacts with elongin $\mathrm{BC}$ and USP7 to modulate the chromatin landscape. Molecular Cell, 64(4), 659-672.

24. Liu, X., Yang, X., Li, Y., Zhao, S., Li, C., Ma, P., \& Mao, B. (2016). Trip12 is an E3 ubiquitin ligase for USP7/HAUSP involved in the DNA damage response. FEBS Letters, 590(23), 4213-4222.

25. Liu, J., Feng, J., Li, L., Lin, L., Ji, J., Lin, C., Liu, L., Zhang, N., Duan, D., Li, Z., Huang, B., Zhang, Y., \& Lu, J. (2020). Arginine methylation-dependent LSD1 stability promotes invasion and metastasis of breast cancer. EMBO Reports, 21(2), e48597.

26. Lu, J., Song, G., Tang, Q., Zou, C., Han, F., Zhao, Z., Yong, B., Yin, J., Xu, H., Xie, X., Kang, T., Lam, Y., Yang, H., Shen, J., \& Wang, J. (2015). IRX1 hypomethylation promotes osteosarcoma metastasis via induction of CXCL14/NF-kappaB signaling. The Journal of Clinical Investigation, 125(5), 1839-1856.

27. Ma, B., Lee, T. L., Hu, B., Li, J., Li, X., Zhao, X., Hou, C., Zhang, C., He, L., Huang, X., Chen, X., Li, J., \& Wu, J. (2019). Molecular characteristics of early-stage female germ cells revealed by RNA sequencing of low-input cells and analysis of genome-wide DNA methylation. DNA Research, 26(2), 105-117.

28. Mascarenhas, M. N., Flaxman, S. R., Boerma, T., Vanderpoel, S., \& Stevens, G. A. (2012). National, regional, and global trends in infertility prevalence since 1990: A systematic analysis of 277 health surveys. PLoS Medicine, 9(12), e1001356.

29. Ohinata, Y., Payer, B., O'Carroll, D., Ancelin, K., Ono, Y., Sano, M., Barton, S. C., Obukhanych, T., Nussenzweig, M., Tarakhovsky, A., Saitou, M., \& Surani, M. A. (2005). Blimp1 is a critical determinant of the germ cell lineage in mice. Nature, 436(7048), 207-213.

30. Palazon-Riquelme, P., Worboys, J. D., Green, J., Valera, A., Martin-Sanchez, F., Pellegrini, C., Brough, D., \& LopezCastejon, G. (2018). USP7 and USP47 deubiquitinases regulate NLRP3 inflammasome activation. EMBO Reports, 19(10), e44766.

31. Peng, Y., Liu, Y., Gao, Y., Yuan, B., Qi, X., Fu, Y., Zhu, Q., Cao, T., Zhang, S., Yin, L., \& Li, X. (2019). USP7 is a novel deubiquitinase sustaining PLK1 protein stability and regulating chromosome alignment in mitosis. Journal of Experimental \& Clinical Cancer Research, 38(1), 468.

32. Rizzino, A., \& Wuebben, E. L. (2016). Sox2/Oct4: A delicately balanced partnership in pluripotent stem cells and embryogenesis. Biochimica et Biophysica Acta, 1859(6), 780-791.

33. Satyaki, P. R., \& Gehring, M. (2017). DNA methylation and imprinting in plants: Machinery and mechanisms. Critical Reviews in Biochemistry and Molecular Biology, 52(2), 163-175.

34. Shan, H., Li, X., Xiao, X., Dai, Y., Huang, J., Song, J., Liu, M., Yang, L., Lei, H., Tong, Y., Zhou, L., Xu, H., \& Wu, Y. (2018). USP7 deubiquitinates and stabilizes NOTCH1 in T-cell acute lymphoblastic leukemia. Signal Transduction and Targeted Therapy, 3, 29.

35. Song, M. S., Salmena, L., Carracedo, A., Egia, A., Lo-Coco, F., Teruya-Feldstein, J., \& Pandolfi, P. P. (2008). The deubiquitinylation and localization of PTEN are regulated by a HAUSP-PML network. Nature, 455(7214), 813-817.

36. Tang, Y., Lv, L., Li, W., Zhang, X., Jiang, Y., Ge, W., \& Zhou, Y. (2017). Protein deubiquitinase USP7 is required for osteogenic differentiation of human adipose-derived stem cells. Stem Cell Research \& Therapy, 8(1), 186.

37. Wang, L., Kumar, S., Dahiya, S., Wang, F., Wu, J., Newick, K., Han, R., Samanta, A., Beier, U. H., Akimova, T., Bhatti, T. R., Nicholson, B., Kodrasov, M. P., Agarwal, S., Sterner, D. E., Gu, W., Weinstock, J., Butt, T. R., Albelda, S. M., \& Hancock, W. W. (2016). Ubiquitin-specific protease-7 inhibition impairs Tip60dependent Foxp3+ T-regulatory cell function and promotes antitumor immunity. EBioMedicine, 13, 99-112.
38. Wang, F., Wang, L., Wu, J., Sokirniy, I., Nguyen, P., Bregnard, T., Weinstock, J., Mattern, M., Bezsonova, I., Hancock, W. W., \& Kumar, S. (2017). Active site-targeted covalent irreversible inhibitors of USP7 impair the functions of Foxp3+ T-regulatory cells by promoting ubiquitination of Tip60. PLoS One, 12(12), e0189744.

39. Wang, Y., Li, Y., Yue, M., Wang, J., Kumar, S., Wechsler-Reya, R. J., Zhang, Z., Ogawa, Y., Kellis, M., Duester, G., \& Zhao, J. C. (2018). N(6)-methyladenosine RNA modification regulates embryonic neural stem cell self-renewal through histone modifications. Nature Neuroscience, 21(2), 195-206.

40. Wang, J., Li, H. Y., \& Su, Z. B. (2020). Stabilization of the histone acetyltransferase Tip60 by deubiquitinating enzyme USP7 stimulates the release of pro-inflammatory mediators in acute lung injury. Journal of Molecular Medicine (Berlin, Germany), 98, 907-921.

41. White, Y. A., Woods, D. C., Takai, Y., Ishihara, O., Seki, H., \& Tilly, J. L. (2012). Oocyte formation by mitotically active germ cells purified from ovaries of reproductive-age women. Nature Medicine, 18(3), 413-421.

42. Wu, C., Xu, B., Li, X., Ma, W., Zhang, P., Chen, X., \& Wu, J. (2017). Tracing and characterizing the development of transplanted female germline stem cells in vivo. Molecular Therapy, 25(6), 1408-1419.

43. Xiao, H., Wen, Y., Pan, Z., Shangguan, Y., Qin, J., Tan, Y., Jiang, H., Li, B., Zhang, Q., Chen, L., \& Wang, H. (2018). Increased $\mathrm{H} 3 \mathrm{~K} 27 \mathrm{ac}$ level of ACE mediates the intergenerational effect of low peak bone mass induced by prenatal dexamethasone exposure in male offspring rats. Cell Death \& Disease, 9(6), 638.

44. Yu, J., Wang, L., Pei, P., Li, X., Wu, J., Qiu, Z., Zhang, J., Ao, R., Wang, S., Zhang, T., \& Xie, J. (2019). Reduced H3K27me3 leads to abnormal Hox gene expression in neural tube defects. Epigenetics \& Chromatin, 12(1), 76.

45. Zagore, L. L., Sweet, T. J., Hannigan, M. M., WeynVanhentenryck, S. M., Jobava, R., Hatzoglou, M., Zhang, C., \& Licatalosi, D. D. (2018). DAZL regulates germ cell survival through a network of polyA-proximal mRNA interactions. Cell Reports, 25(5), 1225-1240 e6.

46. Zhang, C., \& Wu, J. (2016). Production of offspring from a germline stem cell line derived from prepubertal ovaries of germline reporter mice. Molecular Human Reproduction, 22(7), 457-464.

47. Zhang, J., Gao, Q., Li, P., Liu, X., Jia, Y., Wu, W., Li, J., Dong, S., Koseki, H., \& Wong, J. (2011). S phase-dependent interaction with DNMT1 dictates the role of UHRF1 but not UHRF2 in DNA methylation maintenance. Cell Research, 21(12), 1723-1739.

48. Zhang, Z. M., Rothbart, S. B., Allison, D. F., Cai, Q., Harrison, J. S., Li, L., Wang, Y., Strahl, B. D., Wang, G. G., \& Song, J. (2015). An allosteric interaction links USP7 to deubiquitination and chromatin targeting of UHRF1. Cell Reports, 12(9), 1400-1406.

49. Zhang, X. L., Wu, J., Wang, J., Shen, T., Li, H., Lu, J., Gu, Y., Kang, Y., Wong, C. H., Ngan, C. Y., Shao, Z., Wu, J., \& Zhao, X. (2016). Integrative epigenomic analysis reveals unique epigenetic signatures involved in unipotency of mouse female germline stem cells. Genome Biology, 17(1), 162.

50. Zou, K., Yuan, Z., Yang, Z., Luo, H., Sun, K., Zhou, L., Xiang, J., Shi, L., Yu, Q., Zhang, Y., Hou, R., \& Wu, J. (2009). Production of offspring from a germline stem cell line derived from neonatal ovaries. Nature Cell Biology, 11(5), 631-636.

51. Zou, K., Hou, L., Sun, K., Xie, W., \& Wu, J. (2011). Improved efficiency of female germline stem cell purification using fragilis-based magnetic bead sorting. Stem Cells and Development, 20(12), 2197-2204.

52. Zou, K., Wang, J., Bi, H., Zhang, Y., Tian, X., Tian, N., Ma, W., \& Wu, J. (2019). Comparison of different in vitro differentiation conditions for murine female germline stem cells. Cell Proliferation, $52(1), \mathrm{e} 12530$.

Publisher's Note Springer Nature remains neutral with regard to jurisdictional claims in published maps and institutional affiliations. 